\title{
Ionic Liquids: from Neoteric Solvents to New Materials
}

\author{
Montserrat Gómez ${ }^{\mathrm{a}}$, Jean-Christophe Plaquevent ${ }^{\mathrm{b}}$ \\ ${ }^{a}$ Laboratoire Hétérochimie Fondamentale et Appliquée, UMR CNRS 5069, Université de Toulouse 3 - \\ Paul Sabatier, 118 route de Narbonne, 31062 Toulouse cedex 9, France \\ ${ }^{b}$ Université Paul Sabatier, CNRS-UMR 5068, SPCMIB, 118 route de Narbonne, 31062 Toulouse \\ Cedex 9, France \\ gomez@chimie.ups-tlse.fr,plaquevent@chimie.ups-tlse.fr
}

Keywords: ionic liquids.

We are glad and proud to present this special issue of the French Ukrainian Journal of Chemistry, dedicated to the chemistry of ionic liquids.

Ionic liquids (ILs) and their unique properties have given rise to a huge interest in various fields of chemistry. Thanks to their negligible vapor pressure, confinement and recycling are easy. Most of ILs are nonflammable fluids. This, along with their ability to dissolve a large variety of organic and organometallic compounds, as well as the possibility to finely tune their physical and chemical properties, makes them powerful alternative solvents in the context of green chemistry. Moreover it has been shown that they often lead to more efficient and selective processes compared with those in conventional organic solvents, or even to trigger changes on reaction pathways. As known, ILs find applications in many research areas, from new materials to innovative processes, from unprecedented catalytic species to improved extractive methods. Even new routes for pharmaceutical applications were described taking advantage of ILs properties.

In this special issue, composed of nine contributions, some modern aspects of ionic liquids chemistry are summarized, from synthesis to materials, including physicochemical studies, catalysis and chemistry of biomolecules. Santini and co-workers summarize the knowledge about the thermal stability of imidazolium-based ILs. Dez, Glinel and co-workers describe the preparation of ILloaded microreactors based on polyelectrolyte microcapsules. De Viguerie and Marty describe a new family of zwitterionic ILs. An account of Gómez and co-workers review applications of ILs in catalysis, with emphasis on molecular and nanometric metal species, including supported systems. The same group discloses their own studies about palladium nanoparticles in ILs stabilized by mono-phosphines and their catalytic applications. In an account, Plaquevent and co-authors review their results about 
peptide synthesis in ILs. Pollet and Liotta give a comprehensive survey of the reversible process between $\mathrm{CO}_{2}$ and amines. Morel and Trzeciak disclose their studies on the effect of imidazolinium salts bearing hydroxy substituents on palladium-catalysed C-C coupling reactions. A paper of Valade and coworkers deals with the synthesis of nanoparticles of organic conductors in an ionic liquid medium.

This issue takes advantage of different approaches described here above to offer a snapshot of the current trends in Ionic Liquids field. We hope all readers will enjoy the special issue, and that this compilation may give new insights in this promising and exciting area of research. 